\title{
NUEVAS METODOLOGÍAS EDUCATIVAS PARA COMBATIR LA INACTIVIDAD FÍSICA. LA TECNOLOGÍA AL SERVICIO DE LA EDUCACIÓN
}

\author{
Sebastián López Serrano \\ Facultad de Humanidades y Ciencias de la Educación. \\ Dpt. Didáctica de la Expresión M. P. y Corporal \\ Universidad de Jaén \\ sls00013@red.ujaen.es
}

Fecha de Recepción: 22 Marzo 2018

Fecha de Admisión: 10 Abril 2018

\section{RESUMEN}

La sociedad actual se encuentra en constante evolución en busca de nuevas metodologías que permitan una formación integral del alumnado. La evidencia científica actual ha corroborado que una manera de estimular la función cognitiva es a través de la práctica habitual de la actividad física. Se ha demostrado que la inclusión de actividades lúdicas como Exergames (videojuegos con un componente intrínseco de Actividad Física), o nuevas prácticas desarrolladas en educación como la Gamificación, Flipped Classroom o Aprendizaje Basado en Proyectos permiten durante el día académico, aumentar la cantidad diaria de Actividad Física, así como a mejorar la socialización, o el rendimiento cognitivo. Esta sería una fórmula ideal para luchar contra las elevadas tasas de obesidad infantil y juvenil. En España, cerca del 18 \% de los jóvenes son obesos lo cual influye gravemente en problemas no solo en problemas de salud, sino también en problemas sociales y académicos. El objetivo de este trabajo es revisar si la práctica de Exergames influye positivamente en la salud de los usuarios así como posibles beneficios a nivel cognitivo tanto en niños y adolescentes. Además, también se mostrarán algunas aplicaciones prácticas de uso y recomendaciones para su integración en el aula o en el centro educativo.

Palabras clave: exergames; actividad física; cognición; educación

\section{ABSTRACT}

New educational methodologies to combat physical inactivity. Technology at the service of education.

Current society is in constant evolution in search of new methodologies that allow an integral formation of the students. Scientific evidence has confirmed that a way to stimulate cognitive function is through the practice of physical activity. It has been shown that the inclusion of leisure activ- 


\section{NUEVAS METOdOLOGíAS EDUCATIVAS PARA COMBATIR LA INACTIVIDAD FíSICA. LA TECNOLOGÍA AL SERVICIO DE LA EDUCACIÓN}

ities such as exergames (game with an intrinsic component of Physical Activity), or new practices developed in education such as Gamification, Flipped Classroom or Project Based Learning allow during the academic day, increase the daily amount of Physical Activity. Furthermore, they could improve socialization, or cognitive performance. This would be an ideal way to combat high rates of child and adolescent obesity rates. In Spain, about $18 \%$ of young people are obese. This has a serious influence on health, social and academic problems. The aim of this paper is to review whether the practice of exergames influences the health of the users as well as possible cognitive benefits in children and adolescents. In addition, some practical applications of use and recommendations for their integration in the classroom or school will be shown.

Keywords: exergames; physical activity; cognition; education

\section{INTRODUCCIÓN}

Vivimos en una época de incertidumbre y cambio permanente que requiere que utilicemos todos nuestros recursos creativos para poder transformar y adaptar la escuela del siglo XXI a las necesidades actuales. La literatura científica en los últimos años reitera la importancia de incluir en el sistema educativo una metodología de enseñanza afín a la sociedad actual, en la que se incluya el uso de las tecnologías de la información y la comunicación (TIC) puesto que las ventajas que ofrecen son más atractivas que las empleadas en una metodología más tradicional (Sánchez, Ruiz y Sánchez, 2017). Actualmente, podemos encontrar diferentes metodologías que se pueden desarrollar dentro del sistema educativo que garantice el desarrollo de las competencias clave a través del juego. Durante muchos años, se ha considerado al juego como un elemento contrario al proceso de enseñanza-aprendizaje y alejado de la dinámica que ocurría dentro del aula. En este sentido, los videojuegos han sido tildados de elementos distractores y perjudiciales para los usuarios, cuando realmente, no se ha analizado su contenido y en consecuencia, se ha obviado ciertos beneficios asociados a ellos como el desarrollo de habilidades para la resolución de problemas, pensamiento lógico 0 toma de decisiones entre otros. En este caso, podemos destacar la Gamificación, Realidad Aumentada, Flipped Clasroom, Aprendizaje Basado en Proyectos o la creciente tendencia de creación de Scape Room para trabajar los contenidos curriculares. Por otro lado, también podemos destacar algunas herramientas hasta ahora alejadas de la educación y que presentan un gran potencial si se incluyen en estos espacios, como es el caso de los dispositivos móviles o los propios Exergames (EX), entre otros.

Los EX hacen referencia a un formato de videojuego que combina la Actividad Física (AF) y el juego, interpretando los movimientos corporales generando una conexión motriz entre el jugador y el dispositivo. Además, permiten fomentar el aprendizaje mediante desafíos a múltiples niveles de experiencia, favorece las relaciones sociales entre compañeros e incrementa el gasto calórico (Barnett, Cerin y Baranowsky, 2015; Roemmich, Lambiase, McCarthy, Feda y Kozlowski, 2012). También se conocen los beneficios que conlleva la práctica de esta modalidad sobre aspectos cognitivos y académico (Staiano y Calvert, 2011). Cada vez es más creciente que este tipo de actividades se oferten en los recreos 0 al terminar la jornada escolar a modo de actividades complementarias, puesto que los beneficios que ofrecen son muy atractivos para los usuarios. Esta combinación de ejercicio físico y cognitivo puede ser una herramienta fundamental para revertir la inactividad física e incrementar el rendimiento cognitivo y académico del alumnado (Ruiz-Ariza et al., 2017). Según la Organización Mundial de la Salud (OMS), en el año 2010, el 81\% de los jóvenes no realiza el mínimo recomendado de AF, y propone que deben de realizar un mínimo de 60 minutos diarios de AF a una intensidad de moderada a vigorosa.

Las escuelas pueden ser un escenario ideal para brindar a los niños oportunidades para reducir estos porcentajes de inactividad física a pesar de la escasez de tiempo dedicado a la materia de 
Educación Física (EF). Además, debemos destacar el aspecto motivacional y de atracción que los EX desempeñan, incitando al disfrute, promoción de la salud, las relaciones sociales y un adecuado uso de las TIC. A pesar de estos beneficios, Ios estudios son limitados y los efectos de este tipo de ejercicio en la condición física de los alumnados en edad escolar. Serían necesarios realizar más estudios en esta población puesto que es un ambiente propicio para el aprendizaje y desarrollo de hábitos saludables para la vida.

\section{OBJETIVOS}

El presente trabajo se centra en mostrar las diferentes modalidades de EX actuales, su relación con el incremento de los niveles de condición física, y las mejoras derivadas en la cognición. Además, nos permitirá abordar nuevas metodologías educativas en las que los EX presenten un papel activo en la dinámica diaria escolar.

\section{METODOLOGíA}

Se analizaron diferentes artículos de los últimos años que emplearon alguna modalidad de EX en el ámbito escolar. Se escogieron un total de 11 artículos. 5 se centraron en aspectos relacionados con la actividad física mientras que 6 estuvieron centrados en aspectos cognitivos.

\section{Modalidades de Exergames}

El término Exergaming deriva de la combinación de las palabras "exercise" y "gaming", mediante los cuales se pone en común el ejercicio físico y el juego. Es un género relativamente nuevo que presenta una experiencia activa del cuerpo en relación con el dispositivo electrónico. Este género surge a finales de los 80 aunque no es hasta 1998, cuando Konami lanza el juego "Dance Dance Revolution", el cual es considerado uno de los grandes éxitos de esta modalidad. Para su práctica, los jugadores deben de estar subidos a una plataforma y pisar una serie de señales de tal forma que se va desarrollando una coreografía. Otros de los consideramos EX que podemos encontrar sería la videoconsola Nintendo Wii, la cual incluye como principal novedad un mando inalámbrico con sensores de movimiento, gracias al cual los jugadores podían ver reflejado sus gestos en la televisión. Sony también desarrolló un simulador periférico para Play Station 2. Se trata de una cámara que permite al jugador interactuar con el videojuego únicamente mediante movimientos. Por su parte, Microsoft presentó Kinect, un complemento para la videoconsola Xbox 360, el cual permite a los usuarios controlar e interactuar con la consola sin necesidad de un dispositivo controlador de juego. Más recientemente, los Smarthpones o teléfonos inteligentes permiten dar un pequeño paso más allá y ofrecen la posibilidad de desarrollar este tipo de actividades fuera de la casa. Aplicaciones móviles como Zombies, Run! (2012), Ingress (2013) o el fenómeno Pokémon G0 (2016), están consideradas parte de los EX las cuales permiten además incorporar elementos de realidad aumentada. También podemos encontrar otros EX orientados a una práctica de AF más profesional. Por ejemplo, el dispositivo Makoto Arena Training es un juego de fitness que permite entrenamiento mental, Permitiendo a los usuarios el desarrollo de un entrenamiento cardiovascular además de mejorar la relación neurológica entre el cerebro y el cuerpo.

\section{RESULTADOS}

En este trabajo recogemos un total de 11 estudios que han investigado la relación de los EX con aspectos físicos y cognitivos. Como se ha podido observar, los EX se han acreditado como un estereotipo de juego que busca la eliminación de la actividad sedentaria y promoción de un estilo de vida activo. En esta línea, Maddison et al., (2007), en un estudio realizado en adolescentes, reflejaron que el gasto calórico tras la práctica del juego EyeToy de Play Station, se incrementaron los niveles entre 


\section{NUEVAS METOdOLOGíAS EDUCATIVAS PARA COMBATIR LA INACTIVIDAD FíSICA. LA TECNOLOGÍA AL SERVICIO DE LA EDUCACIÓN}

un 129 y $400 \%$. Gao, Chen y Sttoden (2015), tras 36 semanas de intervención mediante Nintendo Wii, mostraron mejoras significativas en comparación con el grupo que practicó AF sin videojuego en la reducción de tiempo sedentario ( $52 \%$ vs $63 \%$; $p$.001) y un mayor nivel de Actividad Física de Moderada a Vigorosa (MVPA) (40\% vs 31\%; $p$ <.001) en niños de entre 6-8 años. En línea anterior, West y Shores (2014), demostraron tras 4 meses de estudio, demostraron una mayor cantidad de MVPA que el grupo que practicó EX ( $M=14.75 \mathrm{~min})$ que para el grupo que continuó con su clase tradicional ( $M=9.5 \mathrm{~min} ; \mathrm{p}<.01)$. Smits-Engelsman, Jelsma y Ferguson, (2016), tras un período de 5 semanas de entrenamiento con la Nintendo Wii, concluyeron que los usuarios que practicaron EX mejoraron su equilibrio, coordinación, resistencia anaeróbica, fuerza y el control de las extremidades inferiores. Por otro lado, Althoff, White y Horvitz, (2016), investigaron los efectos del juego Pokémon Go en cuanto al nivel AF. Dedujeron que el juego de esta modalidad de EX conduce a aumentos significativos del nivel de AF durante un período de 30 días. Proporcionado una mejora de condición física próxima a un $25 \%$ respecto al inicio del juego.

Si nos centramos en aspectos cognitivos, existen evidencias empíricas que indican que los EX por su parte relacionada con la AF, presentan efectos positivos en el rendimiento cognitivo y académico. Por ejemplo, Anderson et al., (2011) llevaron a cabo el segundo estudio piloto de 20 minutos de duración. Un grupo experimental practicó Dance Dance Revolution y el otro Cyber Cycling. En ambos casos, mejoraron las funciones ejecutivas $(p<.001 \mathrm{y}=.03$, respectivamente) respecto al grupo de control que visualizó un video de la misma duración. Best (2012), verificó que los niños que participaron en cuatro sesiones experimentales de Xbox en alta intensidad mejoran las funciones ejecutivas en niños de entre 6 y 10 años ( $p$ <.01). Cada sesión tenía una duración aproximada de $1 \mathrm{~h}$ y se separó de la sesión anterior en un promedio de nueve días. Por su parte, Staiano et al. (2012), experimentaron con la videoconsola Nintendo Wii qué tenía más impacto tras su práctica. Si el juego de manera competitiva (comparan su progreso físico con otros) o el juego de manera cooperativa. Tras 10 semanas de estudio, demostraron que aquellos que practicaron con EX sin importar su condición de juego mejoraron sus funciones ejecutivas respecto al grupo control $(p=$ .018). Además, el grupo de EX competitivo obtuvo mejoras significativas respecto al grupo cooperativo $(p=.020)$. En esta línea, Lisón et al., (2015), comprobaron que 20 minutos de práctica competitiva (Comparando su progreso con los demás) de Xbox Kinect Adventures aumentaba la respuestas psicológicas respecto al grupo de control que solo caminaba $(p<.001)$. Wagener et al. (2012), investigó el impacto del EX Dance Dance Revolution durante 10 semanas. Encontraron que Ios adolescentes que utilizaban este EX mejoraban las relaciones parentales y autoconcepto respecto al grupo control. ( $p=.02$ ). Finalmente, Ruiz-Ariza et al. (2018) analizaron el efecto de 8 semanas del juego para Smartphone Pokémon G0 en el rendimiento cognitivo y la inteligencia emocional en adolescentes. Los jugadores que practicaron Pokémon GO aumentaron su atención selectiva ( $p=$ $.003)$, niveles de concentración $(p>.001)$ y los niveles de sociabilidad $(p=.003)$ respecto a sus compañeros que no lo practicaron.

\section{DISCUSIÓN}

Como hemos podido comprobar, los EX tienen el potencial de reportar beneficios físicos y cognitivos por su relación con la práctica de AF a otras actividades y ámbitos, permitiendo transformar el tiempo sedentario en activo. Peng, Lin y Crouse (2011), realizaron un metanálisis acerca de si los EX se pueden considerar verdaderamente ejercicio y como éstos podrían influir en el gasto calórico, frecuencia cardiaca o el propio consumo de oxígeno. El metanálisis demostró que jugar a los EX produce un aumento significativo de estas variables. Sin embargo, no se encontraron diferencias significativas respecto a la AF realizada de manera tradicional a intensidad moderada. Por su parte, Norris, Hamer \& Stamatakis (2016), en una revisión sistemática que incluía 22 estudios, descubrió 
que las habilidades motoras eran unas de las mayores beneficiadas tras la práctica de los EX. En otra revisión sistemática realizada por Joronen, Aikasalo \& Suvitie (2016), en la que analizaron 10 artículos, se descubrió que los EX tiene algunos efectos positivos sobre el autoconcepto, el interés situacional y la motivación, el disfrute, el bienestar psicológico y social, y diferentes experiencias de aprendizaje. Estos artículos demuestran que la práctica de EX podría aumentar mejorar la condición física y mejorar el bienestar cognitivos de los usuarios.

\section{CONCLUSIONES}

Como se ha podido observar, los EX se han acreditado como un estereotipo de juego que busca la eliminación de la actividad sedentaria y promoción de un estilo de vida activo. Este tipo de videojuegos involucran el movimiento y ayudan a incrementar los niveles de AF lo suficiente como para impactar en la salud y en la condición física. Todo ello, desde una perspectiva lúdica y activa que permite a los niños a tomar conciencia de la importancia de la práctica de AF para su salud así como un adecuado uso de las nuevas tecnologías, sin olvidar que se trata del juego como un elemento para desarrollar del currículo. Al fin y al cabo, los niños utilizan el juego para hacer amigos, superar miedos, resolver problemas y, en general, tomar las riendas de su vida. También se valen de él para practicar y adquirir las destrezas físicas e intelectuales que son fundamentales para el éxito en su cultura, tal y como hemos observado. Afortunadamente, la sociedad actual está consiguiendo desarrollar de manera creativa nuevas herramientas y metodologías que nos permitan enseñar y mejorar las competencias esenciales para el aprendizaje.

\section{REFERENCIAS}

Althoff, T., White, R. W., \& Horvitz, E. (2016). Influence of Pokémon Go on physical activity: Study and implications. Journal of Medical Internet Research, 18(12).

Anderson-Hanley, C., Tureck, K., \& Schneiderman, R. L. (2011). Autism and exergaming: effects on repetitive behaviors and cognition. Psychology research and behavior management, 4, 129.

Barnett, A., Cerin, E., \& Baranowski, T. (2011). Active video games for youth: a systematic review. Journal of Physical Activity and Health, 8(5), 724-737.7

Best, J. R. (2012). Exergaming immediately enhances children's executive function. Developmental psychology, 48(5), 1501.

Gao, Z., Chen, S., \& Stodden, D. F. (2015). A comparison of children's physical activity levels in physical education, recess, and exergaming. Journal of Physical Activity and Health, 12(3), 349354.

Joronen, K., Aikasalo, A., \& Suvitie, A. (2017). Nonphysical effects of exergames on child and adolescent well being: a comprehensive systematic review. Scandinavian journal of caring sciences, 31(3), 449-461.

Lisón, J. F., Cebolla, A., Guixeres, J., Álvarez-Pitti, J., Escobar, P., Bruñó, A., ... \& Baños, R. (2015). Competitive active video games: Physiological and psychological responses in children and adolescents. Paediatrics \& child health, 20(7), 373-376.

Maddison, R., Mhurchu, C. N., Jull, A., Jiang, Y., Prapavessis, H., \& Rodgers, A. (2007). Energy expended playing video console games: an opportunity to increase children's physical activity?. Pediatric exercise science, 19(3), 334-343.

Norris, E., Hamer, M., \& Stamatakis, E. (2016). Active video games in schools and effects on physical activity and health: a systematic review. The Journal of pediatrics, 172, 40-46.

Roemmich, J. N., Lambiase, M. J., McCarthy, T. F., Feda, D. M., \& Kozlowski, K. F. (2012). Autonomy supportive environments and mastery as basic factors to motivate physical activity in children: a controlled laboratory study. International Journal of Behavioral Nutrition and Physical 
Activity, 9(1), 16.

Ruiz-Ariza, A., Casuso, R. A., Suarez-Manzano, S., \& Martínez-López, E. J. (2018). Effect of augmented reality game Pokémon G0 on cognitive performance and emotional intelligence in adolescent young. Computers \& Education, 116, 49-63.

Sánchez Rodríguez, J., Ruiz Palmero, J., \& Sánchez Vega, E. (2014). Las clases invertidas: beneficios y estrategias para su puesta en práctica en la educación superior.

Smits-Engelsman, B. C., Jelsma, L. D., \& Ferguson, G. D. (2016). The effect of exergames on functional strength, anaerobic fitness, balance and agility in children with and without motor coordination difficulties living in low-income communities. Human Movement Science.

Staiano, A. E., \& Calvert, S. L. (2011). Exergames for Physical Education Courses: Physical, Social, and Cognitive Benefits. Child Development Perspectives, 5(2), 93-98.

Wagener, T. L., Fedele, D. A., Mignogna, M. R., Hester, C. N., \& Gillaspy, S. R. (2012). Psychological effects of dance based group exergaming in obese adolescents. Pediatric obesity, 7(5).

West, S. T., \& Shores, K. A. (2014). Does HOPSports promote youth physical activity in physical education classes?. Physical Educator, 71(1), 16. 